\title{
Development of biomaterial surfaces with and without microbial nanosegments
}

\begin{abstract}
Infections by microorganisms are a major problem in public health throughout the world. Artificial materials, including biomedical goods, inherently lack defense against microbial development. Therefore, microbial cells can adhere on any type of artificial surface, particularly in a moist environment, and start to multiply to form a huge population. In this review, we will discuss a strategy for designing antimicrobial polymers and antimicrobial surfaces. Generally, there are five types of antimicrobial polymers: (a) polymeric biocides, (b) biocidal polymers, (c) biocide-releasing polymers, (d) bioactive oligopeptides, and (e) antimicrobial surfaces. Antimicrobial surfaces preventing the growth of microorganisms are a promising method to inhibit the spread of microbial infections. The antimicrobial surfaces can reject the attachment of microbes and/or kill microbes in the vicinity and can be designed to kill microbes on contact. It is recommended that the material surface not release biocidal substances, therefore preventing exhaustion of biocide release to kill microbes. Furthermore, the antimicrobial surfaces are desired to be nontoxic to human cells. The development of contact-active antimicrobial surfaces by grafting antimicrobial nanosegments onto the material surface will be an important topic in the future.
\end{abstract}

Keyword: Antimicrobial; Biomaterial; Nanosegment 